\title{
Prevalence of asymptomatic Plasmodium infections with sub-microscopic parasite densities in the northwestern border of Thailand: a potential threat to malaria elimination
}

Jetsumon Sattabongkot ${ }^{1}$, Chayanut Suansomjit ${ }^{2}$, Wang Nguitragool $^{3}$, Jeeraphat Sirichaisinthop ${ }^{4}$, Saradee Warit ${ }^{5}$, Montip Tiensuwan ${ }^{6}$ and Sureemas Buates ${ }^{2^{*}}$

\begin{abstract}
Background: Asymptomatic infections with sub-microscopic Plasmodium serve as a silent reservoir of disease, critical to sustaining a low level of remanent malaria in the population. These infections must be effectively identified and targeted for elimination. The sensitivity of light microscopy, the traditional method used for diagnosing Plasmodium infections, is frequently insufficient for detecting asymptomatic infections due to the low density of parasitaemia. The objective of this study was to explore the current prevalence of asymptomatic sub-microscopic Plasmodium carriages to evaluate the parasite reservoir amongst residents from 7 hamlets in Tak Province in northwestern Thailand using a highly sensitive molecular method.
\end{abstract}

Methods: Malaria infection was screened in a real-world setting from 3650 finger-prick blood specimens collected in a mass cross-sectional survey using light microscopy and loop-mediated isothermal amplification (LAMP). LAMP results were later confirmed in a laboratory setting in Bangkok using nested PCR, restriction enzyme digestion and DNA sequencing. The association of malaria infection with demographic factors was explored.

Results: Parasite prevalence was $0.27 \%(10 / 3650)$ as determined by microscopy. Sub-microscopic infection prevalence was 2.33\% (85/3650) by LAMP. Of these, 30.6\% (26/85) were infected with Plasmodium falciparum, 52.9\% (45/85) with Plasmodium vivax, 2.4\% (2/85) with Plasmodium malariae, 4.7\% (4/85) with mixed P. falciparum and P. vivax, and 9.4\% (8/85) had parasite densities too low for species identification. Asymptomatic carriages $\left(T<37.5^{\circ} \mathrm{C}\right)$ accounted for $95 \%$ (76/80) of all sub-microscopic cases with the highest prevalence occurring in the subjects $31-45$ years of age $(p \leq 0.035)$. Participants working on plantations or as merchants had an increased infection risk. Evaluation by microscopy identified 10.53\% (10/95) of all Plasmodium infected participants.

Conclusion: Participants carrying asymptomatic Plasmodium infections with sub-microscopic parasite densities are considerable in this area. These findings provide the true disease burden and risk factors in this region. This information helps to direct policy makers towards better schemes and delivery of targeted interventions. Moreover, this is the first study to use LAMP in mass screening for sub-clinical and sub-microscopic infections in a field setting in Thailand. LAMP proves to be a sensitive and field-deployable assay suitable for national malaria control screening campaigns.

Keywords: Prevalence, Asymptomatic, Sub-microscopic, Malaria, Thailand

\footnotetext{
*Correspondence: sbuates@hotmail.com

${ }^{2}$ Department of Microbiology, Faculty of Science, Mahidol University,

Bangkok, Thailand

Full list of author information is available at the end of the article
} 


\section{Background}

Based on the world malaria report in 2017 from 91 countries and areas with ongoing malaria transmission, the progress of malaria control efforts has stalled after an unprecedented session of successfulness in worldwide malaria control [1]. In 2016, there were approximately 216 million malaria cases, an increase of 5 million cases compared to 2015. In 2016, the number of deaths was 445,000 worldwide which is a similar number to the previous year. Of these deaths, $92 \%$ occurred in the African Zone, followed by $6 \%$ in the Southeast Asian Zone, as well as 2\% in the Eastern Mediterranean Zone [1].

According to malaria statistics for Thailand in 2017, malaria control efforts in Thailand have been highly successful, resulting in an $82.2 \%$ decrease in clinical cases over the last 10 years since 2007 from 63,354 to 11,263 cases [2]. Nonetheless, malaria is still endemic in some parts of Thailand. The distribution of malaria in Thailand is patchy and can be typified as 'border malaria' and 'forest malaria', with highest transmission along international borders and in the hills, forests or forest fringes [2]. The borders where malaria outbreaks occurred were along the western border with Myanmar (29.1\%), the southern border with Malaysia (31.3\%), the eastern border with Cambodia (7\%), and to a lesser degree, the eastern border with Laos (2.1\%) [3]. Resultantly, malaria transmission along these four international borders accounted for $69.5 \%$ of the cases of malaria in Thailand [3]. Historically, the western border with Myanmar has had the highest prevalence of malaria in Thailand [2]. This area has been the focus of vigorous malaria control programmes for decades. Consequently, parasite prevalence in this area has declined and the highest parasite prevalence in the country is now in the southern border with Malaysia [3]. Currently, there are 6 Plasmodium species, Plasmodium falciparum, Plasmodium vivax, Plasmodium malariae, Plasmodium ovale curtisi, Plasmodium o. wallikeri as well as Plasmodium knowlesi that can infect humans. Of these, two species, $P$. vivax (76.70\%) and $P$. falciparum (15.23\%) are the predominant species found in Thailand [3].

As the malaria burden still poses serious challenges, the Thai Government has declared a national malaria elimination goal of 2024 [4]. To accomplish this goal, the priorities under the latest strategic plan include increased detection of both symptomatic and asymptomatic malaria together with accurate treatment. Detection of all infected persons, including asymptomatic carriers with low and sub-microscopic parasite densities is critical to the elimination of malaria, as these carriers harbour a parasite reservoir in the population which can still potently be transmitted to mosquitoes and contribute to human transmission [5]. In Thailand, light microscopy analysis of blood smears is the gold standard in malaria diagnosis. However, microscopy is known for being insensitive at low-level parasitaemia. Therefore, the actual prevalence of the malaria parasite should be underestimated. As assessed via standardization with a thick blood film spiked with the parasite, the limit of detection of microscopy for detecting parasitaemia is between 10 and 100 parasites/ $\mu \mathrm{L}(10,000-100,000$ parasites $/ \mathrm{mL}$ ) [6, 7]. Therefore, there is a great need for more accurate methods of detection for community-based studies quantifying asymptomatic and very low-density Plasmodium infections for improving malaria elimination strategies. In addition to being a highly sensitive diagnostic, the ideal method needs to be readily fieldapplicable for pre-elimination field screening teams.

The study site in this current study is located in Tak, a Myanmar-border province, previously the location of Thailand's highest malaria burden. In the past few years, however, this province experienced a substantial decrease in transmission $[8,9]$. However, based on microscopy evaluation, a yearly parasite incidence of 5.04 cases $/ 1000$ was found in 2017. Tak still represents a malaria hotspot in Thailand [3]. For a mass screening survey in this region, most of the research used a quantitative polymerase chain reaction (qPCR) method to detect asymptomatic submicroscopic Plasmodium infections. This method had to be conducted in Bangkok [10, 11]. Up until now, no molecular-based studies have been done in a field setting for mass screening in this area of Thailand.

A newer molecular method, loop-mediated isothermal amplification (LAMP) has been shown to have an estimated detection limit of 1 parasitized red blood cell (RBC)/500 $\mu \mathrm{L}$ of blood [12]. LAMP is a ground-breaking gene amplification technique, and has recently been used in field settings to evaluate malaria prevalence [13-17]. Reverse transcription (RT)-LAMP has been shown to be roughly 100 -fold more sensitive than reverse transcription (RT)-qPCR [18]. LAMP is more accessible in field settings than PCR since it can be deployed outside reference laboratories. LAMP can be performed without a thermal cycler and has diagnostic accuracy comparable to PCR, without demanding complex equipment for sample processing or result analysis. Therefore, LAMP offers a simple, reliable, sensitive, and cost-effective tool for significantly improving the detection of asymptomatic Plasmodium infections with low-density parasites, that otherwise go undetected by microscopy.

To date, there have been no studies done to determine the prevalence of asymptomatic Plasmodium infections with sub-microscopic and low-density malaria reservoirs by LAMP in a real-world field setting of communities in Thailand. Accordingly, a cross-sectional survey was conducted in 3650 people living in malaria-endemic 
communities in Tak Province, Thailand. Here, a malariometric survey performed in the field by LAMP assay to determine the current epidemiology of asymptomatic sub-microscopic carriers was described. This study explored the risk factors associated with residual malaria parasitaemia amongst local residents, for a more comprehensive understanding of the malaria epidemiology.

\section{Methods}

\section{Study site description}

The community-based cross-sectional survey was conducted in Tha Song Yang district, Tak Province, northwestern Thailand (Fig. 1). Tha Song Yang is approximately $595 \mathrm{~km}$ from the capital. It is located at $17^{\circ} 13^{\prime} 36^{\prime \prime} \mathrm{N}$, $98^{\circ} 13^{\prime} 30^{\prime \prime} \mathrm{E}$ with $1010 \mathrm{~m}$ above the sea level. It is situated in the northwest region of Tak, on the Moei River bank near the Myanmar border. The climate is tropical with an average temperature of $26.4{ }^{\circ} \mathrm{C}$. The rainy season is between May and October with an average yearly rainfall of $1540 \mathrm{~mm}$. The inhabitants of this area are approximately $30 \%$ Thai nationality and $70 \%$ ethnic minorities. Normally, there are two peaks of malaria transmission in western Thailand, including Tak Province. One peak is at the beginning of the rainy season (May-August) and the other is at the end of the rainy season (October) [19]. Plasmodium vivax and P. falciparum are predominant in this region, although all human malaria parasites, as well as the simian malaria species P. knowlesi, can be found [20]. There are 6 sub-districts in Tha Song Yan, which are further sub-divided into 56 villages. Among these, 7 hamlets (Nong Bua, Tala Oka, Mae Salid Noi, Suan Oi, Pha Man, Ko Ma Nae, and Orphanage) were chosen for the study to conduct a community-based cross-sectional survey of Plasmodium prevalence.

\section{Data and sample collection}

A mass cross-sectional study was conducted to determine the prevalence of asymptomatic sub-microscopic malaria carriages from 13 to 27 July, 2015. An ethical consent for this study was provided by an ethical committee, Department of Disease Control, Ministry of Public Health, Nonthaburi, Thailand (Ref No 7/54-479). All individuals and all age groups were invited to participate in the study. Signed informed consents were obtained from all participants before enrolment. Interviews were performed with parents and/or legal guardians for participants 7 years or younger. A selection criteria for participation was the absence of anti-malarial drug treatment

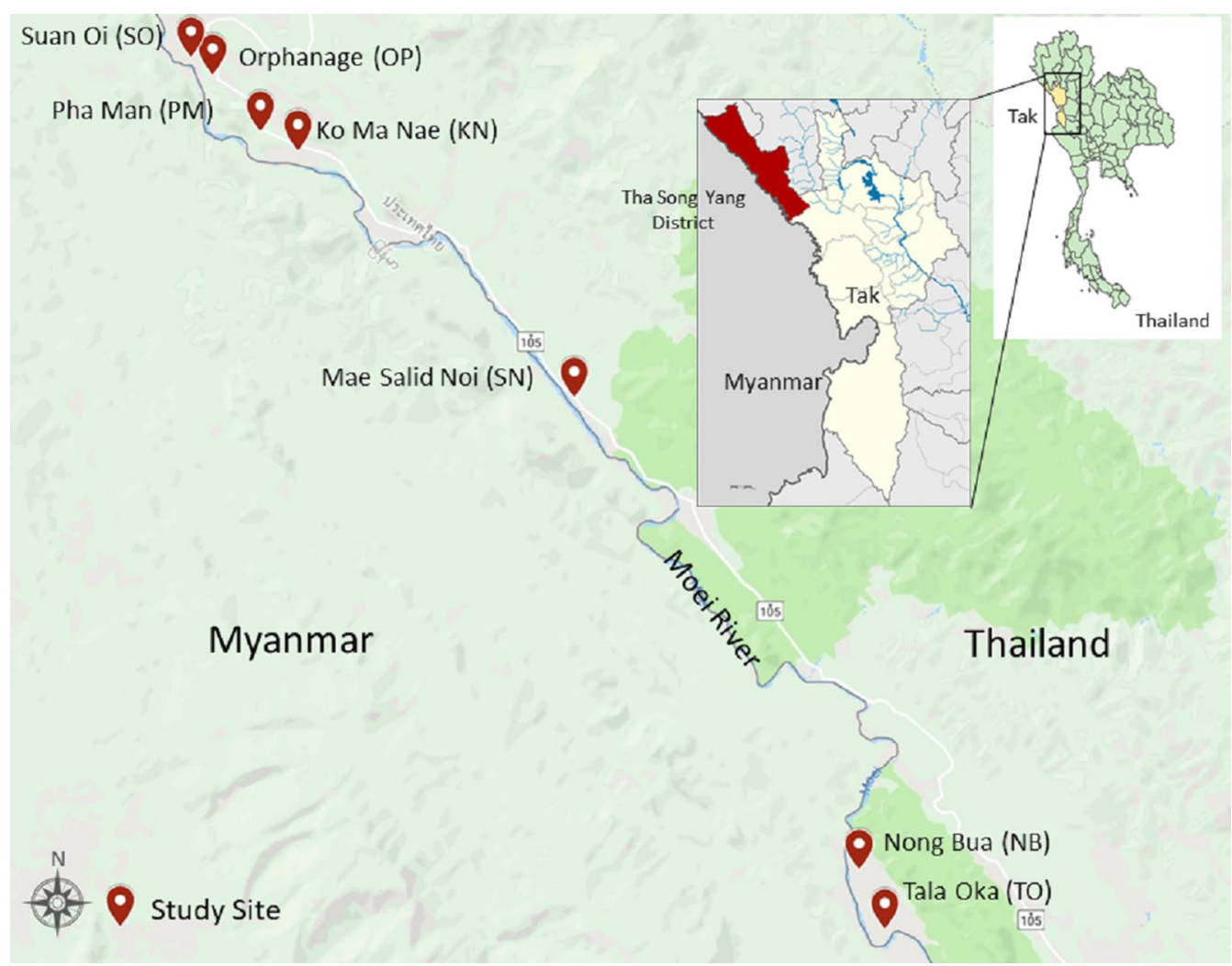

Fig. 1 Study sites in Tha Song Yang district, Tak Province, Thailand 
within the previous 2 weeks. The participant's history of previous malaria infection, body temperature, occupation, age and gender, were recorded at the time of enrolment. The study design for determination of Plasmodium carriages is shown in Fig. 2.

EDTA microtainer tubes were used to collect fingerprick blood samples from 3660 participants who met the inclusion criteria. However, 10 blood samples were excluded from the study since the blood was not sufficient to perform LAMP analysis. For each of the fingerprick blood sample, a portion of the blood was utilized directly to prepare thick and thin blood smears for microscopic analysis. For screening by a Plasmodium genus-specific LAMP assay in the field, $50 \mu \mathrm{L}$ of blood was used. LAMP genus-positive blood samples were further characterized by a LAMP species-specific assay in the field. The remainder of blood samples was kept at $-80{ }^{\circ} \mathrm{C}$ for repeating LAMP analyses in a laboratory setting in Bangkok. Results of the repeated LAMP reactions were later confirmed using a combination of nested
PCR and restriction enzyme digestion, followed by DNA sequencing.

\section{Conventional microscopy}

Thick and Thin blood smear preparation was done on one slide, air-dried, fixed with methanol (thin blood smears) and stained for 10 min with $10 \%$ Giemsa. Thick blood films were examined immediately under a light microscope $(1000 \times$ magnification) by clinical staff in the field as per routine malaria diagnosis. Plasmodium density obtained from thick smear analysis was interpreted as the parasite number detected per 500 white blood cells (WBCs) and was converted into the parasite number $/ \mathrm{ml}$ of blood presuming a WBC count of $8000 \mathrm{WBCs} / \mu \mathrm{L}$ of blood [21]. If no parasites were detected after $500 \mathrm{WBCs}$ were counted, the thick blood smear was classified as negative. The blood smears were re-read in the field by an expert microscopist, of skill level 1 or 2 following the WHO competency assessment protocol, to confirm the result. All microscopists were blinded from the LAMP

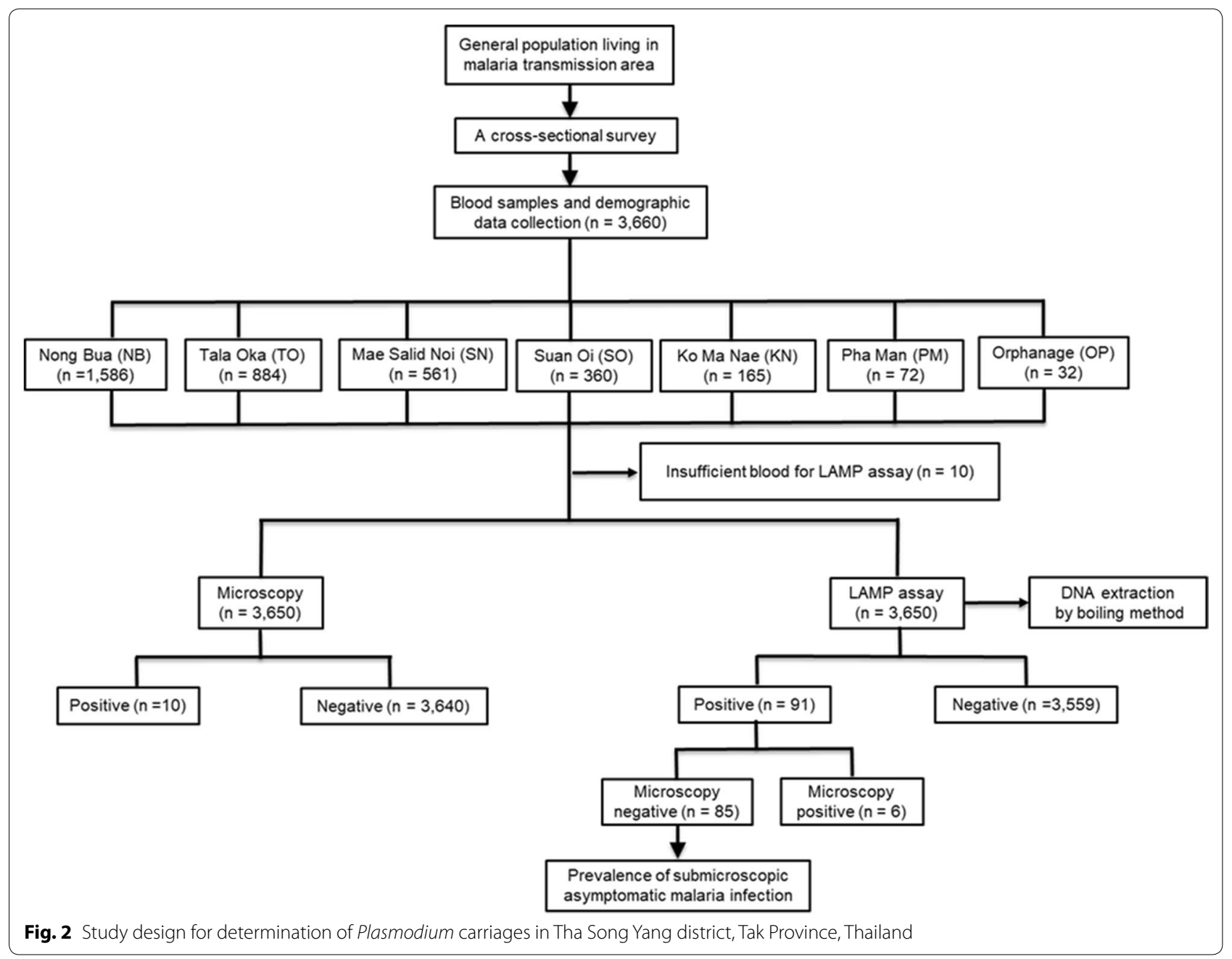


results. Thin blood smears were used for determination of the parasite species via counting the number of parasites detected per 5000 RBCs.

\section{Genomic DNA (gDNA) extraction}

For the LAMP assay, a simple boiling technique was used in this study to extract genomic DNA [16, 17]. Briefly, $50 \mu \mathrm{L}$ of EDTA treated whole blood was added into a $1.5 \mathrm{ml}$-microcentrifuge tube containing $150 \mu \mathrm{L}$ of nuclease-free distilled water, boiled for $5 \mathrm{~min}$ at $95^{\circ} \mathrm{C}$ and centrifuged for $3 \mathrm{~min}$ at $2046 \times g$. For each LAMP reaction, $100 \mu \mathrm{L}$ of the clear supernatant was transferred to a new clean tube and $5.5 \mu \mathrm{L}$ of the supernatant was used immediately. Based on the observation, the supernatant containing gDNA could be kept at $-80^{\circ} \mathrm{C}$ for not more than 7 days without compromising stability.

For nested PCR, DNA extraction was done from frozen EDTA blood samples using a NucleoSpin ${ }^{\circledR}$ Blood mini kit (Macherey-Nagel GmbH, Germany) according to the manufacturer's protocol. For nested PCR analysis, $5 \mu \mathrm{L}$ of the eluted DNA was used directly.

\section{Detection of Plasmodium genus- and species-specific genes by LAMP}

LAMP primer sets targeting $18 \mathrm{~S}$ ribosomal RNA (18srRNA) as described previously by Han et al. [22] were used for detecting all Plasmodium spp. and 4 human Plasmodium parasites including $P$. falciparum, $P$. vivax, $P$. malariae, and $P$. ovale [22]. The primer set used for quantification of the apical membrane antigen-1 (AMA1) gene, which is found during the late schizont stage of all Plasmodium parasites, was used to identify P. knowlesi as described by Lau et al. [23]. A Loopamp DNA amplification kit (Eiken Chemical Co., Ltd., Tokyo, Japan) was used to perform the LAMP reaction. The $25-\mu \mathrm{L}$ LAMP reaction mixture contained $1.6 \mu \mathrm{M}$ of each forward inner primer (FIP) and backward inner primer (BIP) for Plasmodium genus, P. falciparum, $P$. ovale, and $P$. knowlesi and $2.4 \mu \mathrm{M}$ of each FIP and BIP for P. vivax and P. malariae, $0.2 \mu \mathrm{M}$ of each forward outer primer (F3) and backward outer primer (B3), $0.8 \mu \mathrm{M}$ of each loop primer forward (LPF) and loop primer backward (LPB) $12.5 \mu \mathrm{L}$ of $2 \times$ reaction mix, $1 \mu \mathrm{L}$ of Bst DNA polymerase and $5.5 \mu \mathrm{L}$ of DNA template. The LAMP reaction mixture was incubated in a Loopamp real-time turbidimeter (LA-320C, Eiken Chemical Co., Ltd., Tokyo, Japan) or in a water bath for $90 \mathrm{~min}$ at $60^{\circ} \mathrm{C}$ and for $5 \mathrm{~min}$ at $80^{\circ} \mathrm{C}$ for enzyme inactivation. A negative control was included in each run. The LAMP reaction was assessed by the naked eye for endpoint detection or by a Loopamp real-time turbidimeter (LA-320C) for real-time detection. LAMP reactions for Plasmodium genus and species were interpreted as positive when an obvious increase in turbidity was detected by the naked eye or by real-time turbidimeter readings. The results were interpreted as valid when the turbidity was absent in the negative control.

\section{Detection of Plasmodium genus- and species-specific genes by nested PCR}

The species-specific nucleotide sequences of the $18 \mathrm{~S}$ ribosomal RNA (18SrRNA) genes of P. falciparum, $P$. vivax, $P$. malariae, and $P$. ovale were amplified in two successive rounds of PCR as described previously [24]. For the first round of PCR, primers used were specific for all Plasmodium spp. In the second round of PCR, the primers were specific for 4 human malaria species $(P$. falciparum, $P$. vivax, $P$. malariae, and $P$. ovale). The PCR reaction was carried out in a $25-\mu \mathrm{L}$ reaction volume consisting of $12.5 \mu \mathrm{L}$ GoTaq $^{\circledR}$ Green Master Mix (Promega, USA), $0.4 \mu \mathrm{M}$ of each forward and reverse primers for Plasmodium genus, and $5 \mu \mathrm{L}$ of template DNA. In the second round of PCR, the PCR product from the first round was diluted 10 -fold in nuclease-free distilled water, and $5 \mu \mathrm{L}$ was added to a new PCR reaction mixture containing the same concentration of species-specific primers as the genus-specific primers in the first round. For P. knowlesi, 2 sets of primers were used to amplify the small sub-unit ribosomal RNA (ssrRNA) gene. Plasmodium-specific primers ( $r P L U 1$ and $r P L U 5)$ were utilized in the first-round [25]. Plasmodium knowlesi-specific primers (Pmk8 and Pmkr9) were utilized in the second round [26]. Amplification was done in a $25 \mu \mathrm{L}$ reaction mixture containing $5 \mu \mathrm{L}$ of template DNA, $0.25 \mu \mathrm{M}$ of each primer ( $r P L U 1$ and $r P L U 5), 12.5 \mu \mathrm{L}$ of GoTaq $^{\circledR}$ Green Master Mix (Promega, USA). In the first PCR, the ssrRNA gene was amplified following the conditions as described previously [25]. In the second PCR, $5 \mu \mathrm{L}$ of the first PCR product was utilized as the template DNA. The conditions and concentrations of the second PCR were similar to the first one, except the annealing temperature was set at $58{ }^{\circ} \mathrm{C}$ [23]. The amplicons were analysed by electrophoresis in a $2 \%$ agarose gel stained with ethidium bromide and visualized under a UV light.

\section{Confirmation of LAMP products by restriction enzyme digestion}

For further confirmation, LAMP amplified products were validated by restriction enzyme digestion, followed by comparison with the restriction map of the target sequences of each of the LAMP products. For restriction enzyme analysis, DdeI was used for analysis of Plasmodium genus-specific LAMP products. $\mathrm{HpyCH} 4 \mathrm{~V}$ was used for P. falciparum, P. vivax, and P. malariae. AluI was used for $P$. ovale. LAMP products were digested with restriction enzymes at $37{ }^{\circ} \mathrm{C}$ for overnight and were incubated at $65{ }^{\circ} \mathrm{C}$ for $20 \mathrm{~min}$ to inactivate the enzyme. 
Digested LAMP products were analyzed by electrophoresis in a $3 \%$ agarose gel, stained with ethidium bromide and visualized under a UV light.

\section{DNA sequencing}

In order to confirm the Plasmodium genus and species of the LAMP-positive blood samples, the LAMP products were used as template DNA for PCR amplification using LF and LB primers. The products were run on an agarose gel, stained with SYBR safe DNA gel stain (Invitrogen, USA), and the specific band was cut and purified with a QIAquick Gel Extraction kit (Qiagen, Germany). The purified amplicons were cloned into the $\mathrm{pGEM}^{\circledR}$-T Easy Vector (Promega, USA) and sequenced using an ABI3730XL DNA Analyzer (Macrogen, Korea) with 2 universal primers (T7 and SP6). The sequences were aligned with standard sequences of the Plasmodium $18 s r R N A$ gene.

\section{Statistical analysis}

Data analysis of factors associated with Plasmodium infections was done using binary logistic regression. Chi square $\left(\mathrm{X}^{2}\right)$ test with $95 \% \mathrm{CI}$ was used for $p$-value calculation. The SPSS 17.0 statistical software package (SPSS, Inc, Chicago, USA) was used for statistical analysis.

\section{Results}

\section{Study population demographic characteristics}

The mass cross-sectional survey was conducted between 13 and 27 July, 2015. The blood samples were collected with informed consents from 3660 residents living in 7 hamlets in Tha Song Yang district, Tak Province, Thailand. Ten samples had to be excluded from the study since there was insufficient blood to perform a LAMP reaction. Of these 3650 participants, 2035 (55.75\%) were females and 1615 (44.25\%) were males (Table 1). The age of the study population ranged from 1 month to 99 years. Overall, the median age was 15 years ( $\mathrm{IQR}=28$ years) and the mean age was 22.63 years $( \pm \mathrm{SD}=19.34)$. The predominant participants were the age 6-15 years accounted for $34.22 \%(1249 / 3650)$ of all participants. The participants which the age $>60$ years were the minority group accounted for $5.7 \%(208 / 3650)$ of the study population. More than half $(58.68 \%, 2142 / 3650)$ of the participants were foreigners and $41.32 \%(1508 / 3650)$ had Thai citizenship. Participant's body temperature was recorded during blood sample collection. Asymptomatic malaria in this study was classified as a malaria infection found in afebrile participants with axillary temperature $<37.5{ }^{\circ} \mathrm{C}$ at the time of the survey with no presence of fever within the previous 2 days. Participants with body temperature of $<37.5{ }^{\circ} \mathrm{C}$ accounted for $94.83 \%(3411 / 3597)$ of the study population, and only $5.17 \%(186 / 3597)$ had a fever
Table 1 Characteristics of the study population

\begin{tabular}{|c|c|c|}
\hline Characteristics & Number & $\%$ \\
\hline \multicolumn{3}{|l|}{ Study sites $(n=3650)$} \\
\hline Nong Bua & 1579 & 43.26 \\
\hline Tala Oka & 883 & 24.19 \\
\hline Mae Salid Noi & 560 & 15.34 \\
\hline Suan Oi & 359 & 9.84 \\
\hline Ko Ma Nae & 165 & 4.52 \\
\hline Pha Man & 72 & 1.97 \\
\hline Orphanage & 32 & 0.88 \\
\hline \multicolumn{3}{|l|}{ Gender $(n=3650)$} \\
\hline Male & 1615 & 44.25 \\
\hline Female & 2035 & 55.75 \\
\hline \multicolumn{3}{|l|}{ Age } \\
\hline \multicolumn{3}{|l|}{ Mean $=22.63$ years } \\
\hline \multicolumn{3}{|l|}{ Range $=1$ month-99 years } \\
\hline \multicolumn{3}{|l|}{ Median $=15$ years } \\
\hline \multicolumn{3}{|l|}{ $\pm S D=19.34$} \\
\hline \multicolumn{3}{|l|}{$\mathrm{IQR}=28$} \\
\hline \multicolumn{3}{|l|}{ Age $(n=3650)$, years } \\
\hline$\leq 5$ & 636 & 17.42 \\
\hline $6-15$ & 1249 & 34.22 \\
\hline $16-30$ & 640 & 17.53 \\
\hline $31-45$ & 579 & 15.86 \\
\hline $46-60$ & 338 & 9.26 \\
\hline$>60$ & 208 & 5.70 \\
\hline \multicolumn{3}{|l|}{ Body temperature $(n=3597),{ }^{\circ} \mathrm{C}$} \\
\hline$\geq 37.5$ & 186 & 5.17 \\
\hline$<37.5$ & 3411 & 94.83 \\
\hline Not determine $e^{a}$ & 53 & \\
\hline \multicolumn{3}{|l|}{ Citizenship $(n=3650)$} \\
\hline Thai citizenship & 1508 & 41.32 \\
\hline Foreigner & 2142 & 58.68 \\
\hline \multicolumn{3}{|l|}{ Occupation ( $n=3650$ ) } \\
\hline Student & 1440 & 39.45 \\
\hline Child & 633 & 17.34 \\
\hline Hireling/labour & 781 & 21.40 \\
\hline Plantation & 493 & 13.51 \\
\hline Housekeeper/housewife & 74 & 2.03 \\
\hline Merchant & 66 & 1.81 \\
\hline $\begin{array}{l}\text { Other (e.g., cripple, retired, monk, govern- } \\
\text { ment officer) }\end{array}$ & 163 & 4.47 \\
\hline
\end{tabular}

a 53 samples were excluded since body temperature data was not available

$\left(\geq 37.5^{\circ} \mathrm{C}\right)$. Three febrile participants were blood smear negative but were later found to be LAMP positive. Using LAMP, one febrile participant (a foreigner) with a body temperature of $38^{\circ} \mathrm{C}$ had $P$. vivax infection. The other 2 participants with body temperature of $38.1^{\circ} \mathrm{C}$ (Thai) and $39^{\circ} \mathrm{C}$ (foreigner) had $P$. vivax infection and $P$. falciparum infection, respectively. The most frequent occupation 
among participants $(39.45 \%, 1440 / 3650)$ was student. Among the 7 study sites, Nong Bua had the highest population number $(43.26 \%, 1579 / 3650)$ followed by Tala Oka, Mae Salid Noi, Suan Oi, Ko Ma Nae, Pha Man, and Orphanage, respectively.

\section{LAMP and microscopy-based determination of Plasmodium carriages}

Using LAMP, a total of 3650 blood samples were screened in the field. For LAMP genus-specific screening of sub-microscopic malaria infections, $50 \mu \mathrm{L}$ of each finger prick blood sample was used directly for DNA extraction by a boiling method and $5.5 \mu \mathrm{L}$ of freshly prepared supernatant was used immediately. Approximately $200 \mu \mathrm{L}$ of the remaining blood was kept at $4{ }^{\circ} \mathrm{C}$ while waiting for the LAMP genus-specific results. For LAMP genus-positive samples, the supernatants were kept at $-80^{\circ} \mathrm{C}$ for overnight before being

Table 2 Detailed comparison of LAMP and nested PCR combined with restriction enzyme digestion for Plasmodium identifications

Parasite(s) detected by each method ${ }^{a}$ (no. of samples; $n=3650$ blood samples)

\begin{tabular}{ll}
\hline LAMP & $\begin{array}{l}\text { Nested PCR combined } \\
\text { with RE digestion of LAMP } \\
\text { products }^{\mathbf{b}}\end{array}$ \\
\hline P. falciparum (27) & P. falciparum (26), negative (1) \\
P. vivax (47) & P. vivax (45), negative (2) \\
P. falciparum + P. vivax (4) & P. falciparum + P. vivax (4) \\
P. malariae (2) & P. malariae (2) \\
Plasmodium spp. ${ }^{\text {e }}$ (8) & Plasmodium spp. (8) \\
Negative (3562) & Negative (3562) \\
\hline
\end{tabular}

a Each row displays the results obtained from identical blood samples. Discordant results between LAMP and nested PCR combined with restriction enzyme digestion are showed in italicface

b Genus and species-specific LAMP products were digested with restriction enzyme to confirm the specificity of LAMP products

c Positive for $P$. falciparum by LAMP but negative after restriction enzyme digestion of LAMP products

d Positive for $P$. vivax by LAMP but negative after restriction enzyme digestion of LAMP products

e Positive for genus-specific LAMP but negative for species-specific LAMP used for LAMP species-specific analysis. For samples deemed positive in the genus-specific LAMP assay or both genus- and species-specific LAMP assays, $200 \mu \mathrm{L}$ of the remaining blood samples was kept at $-80{ }^{\circ} \mathrm{C}$ for LAMP confirmation in the laboratory.

Of the 3650 blood samples screened in the field, LAMP successfully amplified Plasmodium DNA in 2.58\% (94/3650). Among these LAMP-positive samples, $0.16 \%(6 / 3650)$ were also positive by microscopy. Thus, LAMP identified a substantially higher number of Plasmodium carriages $(2.49 \% ; 88 / 3650)$ as shown in Table 2. LAMP results in the field were later repeated in a laboratory setting in Bangkok, and were confirmed using a combination of nested PCR, restriction enzyme digestion, and DNA sequencing. After confirmation of 88 LAMP-positive samples, there were 3 discordant results composed of 1 sample positive for $P$. falciparum and 2 samples positive for $P$. vivax by LAMP. These samples were negative by restriction enzyme digestion. In summary, overall the prevalence of sub-microscopic infections as determined by LAMP was $2.33 \%$ (85/3650). Details of the prevalence of sub-microscopic Plasmodium infections detected by LAMP in the 7 hamlets are presented in Table 3.

Thick and thin blood films from the 3650 blood samples were analysed by Giemsa-stained smear examination by local staff in the field and later confirmed by an expert microscopist. After the initial and second round of microscopy, only 10 samples were found positive for Plasmodium infections (3 in Suan Oi, 2 in Nong Bua and Orphanage, and 1 in Mae Salid Noi, Ko Ma Nae, and Pha Man), representing an overall prevalence of $0.27 \%(10 / 3650)$ (Table 3). These 10 samples classified as positive by microscopy originated from 8 male and 2 female subjects. Among 10 samples positive by microscopy, 4 samples were negative by LAMP.

As shown in Table 4, the majority of sub-microscopic Plasmodium carriages was a single $P$. vivax infection, accounting for $52.9 \%(45 / 85)$ of the cases. A single $P$. falciparum infection was present in $30.6 \%(26 / 85)$ of the subjects. A single $P$. malariae infection accounted for $2.35 \%(2 / 85)$ of the subjects. Mixed infections between P. falciparum and P. vivax accounted for $4.75 \%$

Table 3 Prevalence of Plasmodium carriages based on microscopy and LAMP

\begin{tabular}{|c|c|c|c|c|c|c|c|c|}
\hline Hamlet & Nong Bua & Tala Oka & Mae Salid Noi & Suan Oi & Ko Ma Nae & Pha Man & Orpha-nage & Total \\
\hline Total population (n) & 1579 & 883 & 560 & 359 & 165 & 72 & 32 & 3650 \\
\hline Microscopy, n (\%) & $2^{\mathrm{a}}(0.13 \%)$ & $0(0 \%)$ & $1(0.18 \%)$ & $3^{b}(0.84 \%)$ & $1(0.61 \%)$ & $1(1.39 \%)$ & $2(6.25 \%)$ & $10(0.27 \%)$ \\
\hline LAMP, n (\%) & $49(3.1 \%)$ & $12(1.36 \%)$ & $5(0.89 \%)$ & $21(5.85 \%)$ & $1(0.61 \%)$ & $1(1.39 \%)$ & $2(6.25 \%)$ & $91(2.49 \%)$ \\
\hline Sub-microscopy, n (\%) & 49 (3.1\%) & $12(1.36 \%)$ & $4(0.71 \%)$ & $20(5.57 \%)$ & $0(0 \%)$ & $0(0 \%)$ & $0(0 \%)$ & $85(2.33 \%)$ \\
\hline
\end{tabular}

a Both samples were positive by microscopy but negative by LAMP

b Only one sample was positive by microscopy and LAMP 
Table 4 Details of sub-microscopic Plasmodium species

\begin{tabular}{lllllllll}
\hline Hamlet & Nong Bua & Tala Oka & Mae Salid Noi & Suan Oi & Ko Ma Nae & Pha Man & Orpha-nage & Total \\
\hline Total population (n) & 1579 & 883 & 560 & 359 & 165 & 72 & 32 & 3650 \\
Sub-microscopy n (\%) & $49(3.1 \%)$ & $12(1.36 \%)$ & $4(0.71 \%)$ & $20(5.57 \%)$ & $0(0 \%)$ & $0(0 \%)$ & $0(0 \%)$ & $85(2.33 \%)$ \\
Plasmodium spp. & & & & & & & \\
PV & $22(44.9 \%)$ & $8(66.67 \%)$ & $1(25 \%)$ & $14(70 \%)$ & $0(0 \%)$ & $0(0 \%)$ & $0(0 \%)$ & $45(52.9 \%)$ \\
Pf & $17(34.7 \%)$ & $3(25 \%)$ & $3(75 \%)$ & $3(15 \%)$ & $0(0 \%)$ & $0(0 \%)$ & $0(0 \%)$ & $26(30.6 \%)$ \\
Pm & $2(4.08 \%)$ & $0(0 \%)$ & $0(0 \%)$ & $0(0 \%)$ & $0(0 \%)$ & $0(0 \%)$ & $0(0 \%)$ & $2(2.35 \%)$ \\
Pf+PV & $4(8.16 \%)$ & $0(0 \%)$ & $0(0 \%)$ & $0(0 \%)$ & $0(0 \%)$ & $0(0 \%)$ & $0(0 \%)$ & $4(4.75 \%)$ \\
P. spp. & $4(8.16 \%)$ & $1(8.33 \%)$ & $0(0 \%)$ & $3(15 \%)$ & $0(0 \%)$ & $0(0 \%)$ & $0(0 \%)$ & $8(9.41 \%)$ \\
\hline
\end{tabular}

Plasmodium. spp.: Plasmodium genus positive but species negative

$P v$, P. vivax; Pf, P. falciparum; Pm, P. malariae

$(4 / 85)$ of the cases and $9.41 \%(8 / 85)$ of the cases identified as Plasmodium genus-positive.

\section{Demographic characteristics of the study population associated with sub-microscopic Plasmodium infections}

Detailed analysis of the association of the demographic characteristics of the study population and sub-microscopic Plasmodium infections is shown in Table 5. Although the prevalence of sub-microscopic malaria infections was higher in female (2.6\%) than male (1.98\%) subjects, no significant difference was observed between these two groups. Most LAMP-positive sub-microscopic Plasmodium infections were found in adults and were significantly higher in subjects aged $31-45$ years $(3.28 \%, 19 / 579) p \leq 0.035$. Among the positive samples, the youngest and oldest subjects were 1 and 78 years of age, respectively. Most of the sub-microscopic malaria infections $(95 \%, 76 / 80)$ were asymptomatic, since indications of malaria-like symptoms, such as headache, chills and fever were not observed. The majority of the sub-microscopic cases of malaria occurred in the participants with no Thai citizenship $(60 \%, 51 / 85)$. Furthermore, a strong association between asymptomatic Plasmodium infections and patient occupations was observed in this study. The risk of acquiring asymptomatic sub-microscopic Plasmodium infections significantly increased in individuals working on the plantation $(p \leq 0.013)$ or as merchants $(p \leq 0.015)$. The odds ratios of plantation and merchant occupations were approximately 2 and 4 times greater than other occupations, respectively. Among 7 hamlets, the prevalence of asymptomatic Plasmodium infections was significantly high in Nong Bua $(3.1 \%$; 49/1579) $p \leq 0.004$ and Suan Oi $(5.57 \%$; 20/359) $p \leq 0.05$.

\section{Discussion}

In 2016, Thailand had approximately 11,522 malaria cases, or 0.17 cases/people [27], and was considered a region of low malaria transmission. Currently, the country is in 'control' phase and proposes to achieve malaria elimination by the year 2024 based on World Health Organization efforts, and the National Malaria Strategy [28]. This is a part of a wider goal for a malaria-free AsiaPacific by the year 2030, proclaimed by the Asia-Pacific Leaders Malaria Alliance at the 9th East Asia Summit in 2014. To reach such a goal, rapid identification (including parasite speciation) of asymptomatic and symptomatic individuals as well as an accurate and proper treatment of symptomatic infections and malaria reservoirs are critical to accomplish. Individuals with asymptomatic submicroscopic malaria infections are silent reservoirs of the parasite and pose a serious challenge to disease control efforts due to their ability to maintain transmission within the population at a low level.

The studied area in this current study is one of the most malaria-endemic regions of Thailand. Although there has been a marked reduction in malaria transmission, Tak Province in 2017 was still classified as a high transmission area based on the WHO 2016 index, with an estimated annual incidence of 5.04 cases/1000 people [27]. In 2017, an estimated annual incidence in Thailand was 0.17 cases $/ 1000$. The estimated annual incidence in Tak Province was approximately 29 times the national average [3]. This estimate in incidence was obtained by the standard malaria diagnosis method of light microscopy analysis of blood smears which lacks sensitivity enough for detecting low-level parasitaemia [6]. To get the actual number of malaria incidence in Thailand, molecular techniques which are more sensitive have been used as an alternative method.

Quantitative polymerase chain reaction (qPCR) has been used to detect a number of asymptomatic submicroscopic malaria infections in this study area $[10,11]$. 
Table 5 Demographic characteristics of the study population associated with asymptomatic sub-microscopic Plasmodium infections

\begin{tabular}{|c|c|c|c|c|}
\hline Characteristics & Total samples & $\begin{array}{l}\text { Prevalence of sub-microscopic } \\
\text { infections } s^{\mathrm{a}}, \mathrm{n}(\%)\end{array}$ & Odds Ratio $(95 \% \mathrm{Cl})$ & P value $^{\text {b }}$ \\
\hline \multicolumn{5}{|l|}{ Gender } \\
\hline Male & 1615 & $32(1.98 \%)$ & $1.32(0.85-2.05)$ & 0.223 \\
\hline Female & 2035 & $53(2.6 \%)$ & & \\
\hline \multicolumn{5}{|l|}{ Age (years) } \\
\hline$\leq 5$ & 636 & $9(1.42 \%)$ & Ref. group & - \\
\hline $6-15$ & 1249 & $25(2 \%)$ & $1.43(0.66-3.07)$ & 0.366 \\
\hline $16-30$ & 640 & $18(2.81 \%)$ & $2.02(0.9-4.54)$ & 0.087 \\
\hline $31-45$ & 579 & $19(3.28 \%)$ & $2.36(1.06-5.27)$ & $0.035^{*}$ \\
\hline $46-60$ & 338 & $9(2.66 \%)$ & $1.91(0.75-4.85)$ & 0.175 \\
\hline$>60$ & 208 & $5(2.4 \%)$ & $1.71(0.57-5.17)$ & 0.339 \\
\hline \multicolumn{5}{|l|}{ Body temperature ${ }^{\mathrm{C}}\left({ }^{\circ} \mathrm{C}\right)$} \\
\hline$\geq 37.5$ & 186 & $4(2.15 \%)$ & $0.96(0.35-2.66)$ & 0.94 \\
\hline$<37.5$ & 3411 & $76(2.23 \%)$ & & \\
\hline \multicolumn{5}{|l|}{ Citizenship } \\
\hline Thai citizenship & 1508 & $34(2.25 \%)$ & $1.06(0.68-1.64)$ & 0.795 \\
\hline Foreigner & 2142 & $51(2.38 \%)$ & & \\
\hline \multicolumn{5}{|l|}{ Study site } \\
\hline Nong Bua & 1579 & 49 (3.1\%) & $4.45(1.59-12.38)$ & $0.004^{*}$ \\
\hline Tala Oka & 883 & $12(1.36 \%)$ & $1.91(0.61-5.96)$ & 0.264 \\
\hline Mae Salid Noi & 560 & $4(0.71 \%)$ & Ref. group & - \\
\hline Suan Oi & 359 & $20(5.57 \%)$ & $8.26(2.79-24.37)$ & $0^{*}$ \\
\hline Ko Ma Nae & 165 & $0(0 \%)$ & 0 & 1 \\
\hline Pha Man & 72 & $0(0 \%)$ & 0 & 1 \\
\hline Orphanage & 32 & $0(0 \%)$ & 0 & 1 \\
\hline \multicolumn{5}{|l|}{ Occupation } \\
\hline Student & 1440 & $32(2.22 \%)$ & $1.58(0.75-3.33)$ & 0.23 \\
\hline Hireling/labour & 781 & $18(2.32 \%)$ & $1.64(0.73-3.68)$ & 0.229 \\
\hline Child & 633 & $9(1.42 \%)$ & Ref. group & - \\
\hline Plantation & 493 & 19 (3.85\%) & $2.78(1.24-6.19)$ & $0.013^{*}$ \\
\hline Housekeeper & 74 & $0(0 \%)$ & 0 & 1 \\
\hline Merchant & 66 & $4(6.06 \%)$ & $4.47(1.34-14.92)$ & $0.015^{*}$ \\
\hline $\begin{array}{l}\text { Other (e.g., cripple, retired, monk, } \\
\text { government officer) }\end{array}$ & 163 & $3(1.84 \%)$ & $1.29(0.35-4.85)$ & 0.698 \\
\hline
\end{tabular}

*Statistically significant difference

a Sub-microscopic infection was defined when the sample was LAMP-positive but microscopy negative

b $P$ values were obtained by binary logistic regression and is significant at $<0.05$

c 53 samples were excluded since the body temperature data was not available

Although this assay is sensitive, it must be performed in a laboratory setting. qPCR is not applicable for use in the field. To date, no molecular methods were conducted in real-life field settings for the malaria mass screening in this area of Thailand. The current study is the first mass cross-sectional survey of sub-clinical and sub-microscopic Plasmodium infections in Thailand conducted in field clinics using a LAMP method. LAMP results revealed that there were 3 samples positive by LAMP but negative after LAMP product confirmation by restriction enzymes digestion. These false positives could be due to haemolysis or high levels of lipid in the plasma of the blood samples. Moreover, 4 samples were positive by microscopy but negative by LAMP. In these samples, the parasite density ranged between 16 and 1440 parasites $/ \mu \mathrm{L}$, which could be detected by LAMP. Thus, there should be other causes for obtaining LAMP-negative results including a lower efficiency of template DNA 
preparation or DNA degradation during the preparation process. Overall, the LAMP method performed in the real-world field setting demonstrated reliable results. In this study, LAMP proves to be easy to operate and simple to adapt for use in any environment and field condition, and can be used as a reliable method for discerning the hidden malaria reservoir in various communities.

This current study found that malaria infection rates estimated by LAMP in the mass blood survey of this community were 8.6 times higher than those estimated by an expert microscopist. At the time of this survey, the prevalence of Plasmodium infections was 2.6\% (95/3650) and over $85.6 \%$ were asymptomatic sub-microscopic infections. This suggests that there is a substantial proportion of Plasmodium-infected individuals with both asymptomatic and sub-microscopic densities in this area. This current finding is in agreement with the results from the previous study by Baum et al. conducted in the same area using qPCR, which found that the prevalence of asymptomatic and sub-microscopic Plasmodium infections in the population was 3.0 and $90.2 \%$, respectively [20]. In Baum's study, individuals with suspected malaria from the malaria clinic and hospital were included. This may explain why the infection rate determined by Baum et al. was slightly higher than in this current study. However, while highly sensitive, LAMP itself has a limit of detection. Therefore, the number of sub-microscopic infections could be higher than reported in this current finding. If LAMP targets a gene with a higher copy number compared to the $18 \mathrm{~S}$ ribosomal RNA (18srRNA) gene used in this study, this limit of detection may be decreased making a LAMP assay to be more sensitive.

Amongst the low-density asymptomatic cases in this study, the most prevalent species was $P$. vivax. This malaria species distribution is similar to that reported in Imwong's study conducted in the northwestern border of Thailand [11] and Nguitragool's study performed in Kanchanaburi and Ratchaburi Provinces in western Thailand [29]. The similarity likely indicates the homogeneity of the distribution of Plasmodium species in western Thailand.

Moreover, this current study also observed evidence of heterogeneity of Plasmodium prevalence of asymptomatic malaria infections, with some hamlets being completely free of infections, while in other nearby hamlets separated by short distances, approximately $5.57 \%$ of the hamlet population was infected. The heterogeneity in the prevalence of malaria infections in this study is in agreement with a previous finding performed in southern Laos PDR [30] and in western Cambodia [31]. Malaria prevalence with spatial heterogeneity between nearby hamlets showed an important variability in Plasmodium epidemiology at the community level. The heterogeneity in the Plasmodium infection prevalence may at least in part be described by environmental factors, including abundance of mosquito larval habitats, mosquito densities, house location in relation to mosquito breeding grounds, house construction materials and design, and resident protective measures and exposure to mosquitoes due to occupations. It appears that the hamlet with the infected population is a local transmission hotspot that needs special attention by the malaria control programmes.

The protective immunity in malaria-endemic areas correlates with age, which is considered one of the most important factors [32]. Generally, young children are the most susceptible to developing the disease. However, older children and adults are more likely to harbour asymptomatic infections since they might have had several episodes of Plasmodium infections and acquired immunity [33,34]. This current study found that the peak risk for low-density asymptomatic Plasmodium infections was in the age group of 31-45 years. A similar peak prevalence in adults has recently been reported in eastern Myanmar which found that all asymptomatic infections were restricted to participants aged $>17$ years [35]. This indicates that the infections were more likely transmitted outside of residential areas and younger individuals may have received less vector exposure. Older individuals may have developed enough immune response to suppress the infection allowing it to persist for a period of time without causing any symptoms.

Previously, the study by Zaw et al. conducted in eastern Myanmar found that there was a statistically significant difference in infection rates among occupations. This previous study showed that much higher infection rates were observed in men working in the forests [35]. Consistent with Zaw's study, a risk factor associated with forest-related activities was observed in this current study since individuals who work on the plantation had significantly increased risk of having asymptomatic sub-microscopic Plasmodium infections. Interestingly, a significantly increased risk was also found in merchants, which is consistent with the correlation with extent of outdoor-related activities. Moreover, many past findings have observed that males are more likely to get infected by malaria than females [14, 30, 35]. However, no gender imbalance was observed in this present study. Both males and females are likely to have the same risk of contracting malaria.

In this study, the LAMP malaria assay provides a promising tool for being used in remote field settings, which could render increased efficiency of ongoing malaria control interventions. However, there are some limitations. The false positive could be occurred when using the supernatant, which was not completely clear from the cell pellet especially in blood samples with haemolysis or 
with high level of lipid in the plasma. The supernatant should be cleared by centrifugation twice before being used. The turbidimetric determination of the LAMP reaction by visual observation is sometimes difficult especially in the weak positive result. The false negative results of LAMP assay could be observed when using the supernatant, which was repeated freezing and thawing or kept on ice for more than 4-5 h. The supernatant should be immediately used to prevent DNA degradation.

\section{Conclusion}

These current findings based on the LAMP survey in a real-world fielding setting reveal substantial sub-microscopic Plasmodium carriages among asymptomatic individuals in Tak Province in northwestern Thailand. This study indicates that light microscopy, a rountinely used diagnostic technique, excessively underestimates true malaria parasite prevalence. Thus, it is imperative to employ a higher sensitivity method such as LAMP for parasite field detection in the phase of malaria elimination. These findings also provide an understanding of the current status of asymptomatic sub-microscopic Plasmodium infection prevalence, and determine the key demographic characteristics of Plasmodium infected cases, such as age groups and occupations. This knowledge could provide crucial information regarding potential infection sources, and could assist in determining the risk of infection within individual hamlets. These findings have important practical implications for targeting activities to eliminate malaria including mass screening and mass anti-malarial drug administration.

\section{Abbreviations}

LAMP: loop-mediated isothermal amplification; RT-LAMP: reverse transcription- LAMP.

\section{Authors' contributions}

JS contributed to study design. CS performed all experiments. WN and JS collected blood samples and donor symptom history. SW performed cloning for DNA sequencing. MT involved in statistical analysis. SB conceived the study and wrote the manuscript. All authors read and approved the final manuscript.

\section{Author details \\ ${ }^{1}$ Mahidol Vivax Research Unit, Faculty of Tropical Medicine, Mahidol University, Bangkok, Thailand. ${ }^{2}$ Department of Microbiology, Faculty of Science, Mahidol University, Bangkok, Thailand. ${ }^{3}$ Department of Molecular Tropical Medicine and Genetics, Faculty of Tropical Medicine, Mahidol University, Bangkok, Thailand. ${ }^{4}$ Bureau of Vector Borne Diseases, Pra Phuttabhat, Saraburi, Thailand. ${ }^{5}$ Tuberculosis Research Laboratory, Medical Molecular Biology Research Unit, BIOTEC, National Science and Technology Development Agency, Thailand Science Park, Pathum Thani, Thailand. ${ }^{6}$ Department of Mathematics, Faculty of Science, Mahidol University, Bangkok, Thailand.}

\section{Acknowledgements}

We would like to acknowledge assistance with data collection by the staff of the Department of Public Health in Tha Song Yang District, Tak Province, Thailand. We also thank the staff of Mahidol Vivax Research Unit, Faculty of Tropical Medicine, Mahidol University, Bangkok, Thailand for technical support; the staff of the malaria clinic in Mae Sot District, Tak Province, Thailand for their support in blood sample collection and microscopic examination.

\section{Competing interests}

The authors declare that they have no competing interests.

\section{Availability of data and materials}

The data supporting the conclusion of this article are included within the article. Raw data are available from the corresponding author upon request.

\section{Consent for publication}

Not applicable.

\section{Ethics approval and consent to participate}

This study was approved by ethical committee of the Department of Disease Control, Ministry of Public Health, Nonthaburi, Thailand (Ref No 7/54-479). Signed informed consents were obtained from all participants before enrollment.

\section{Funding}

Not applicable.

\section{Publisher's Note}

Springer Nature remains neutral with regard to jurisdictional claims in published maps and institutional affiliations.

Received: 28 June 2018 Accepted: 4 September 2018

Published online: 12 September 2018

\section{References}

1. WHO. World malaria report 2017. Geneva: World Health Organization; 2017.

2. Bureau of Epidemiology and Bureau of Vector-borne Diseases. Annual epidemiological surveillance report 2017. Nonthaburi: Ministry of Public Health; 2017.

3. Bureau of Vector-Borne Diseases. Annual Report Malaria situation in Thailand 2017. Nonthaburi: Ministry of Public Health; 2017.

4. Bureau of Vector-Borne Disease. National Strategic Plan for Malaria Control and Elimination in Thailand 2017-2026. Nonthaburi: Ministry of Public Health; 2017.

5. Tadesse FG, Slater HC, Chali W, Teelen K, Lanke K, Belachew M, et al. The relative contribution of symptomatic and asymptomatic Plasmodium vivax and Plasmodium falciparum infections to the infectious reservoir in a low-endemic setting in Ethiopia. Clin Infect Dis. 2018;66:1883-91.

6. Wongsrichanalai C, Barcus MJ, Muth S, Sutamihardja A, Wernsdorfer WH. A review of malaria diagnostic tools: microscopy and rapid diagnostic test (RDT). Am J Trop Med Hyg. 2007;77:119-27.

7. Wu L, van den Hoogen LL, Slater H, Walker PG, Ghani AC, Drakeley CJ, et al. Comparison of diagnostics for the detection of asymptomatic Plasmodium falciparum infections to inform control and elimination strategies. Nature. 2015;528:S86-93.

8. Carrara VI, Sirilak S, Thonglairuam J, Rojanawatsirivet C, Proux S, Gilbos V, et al. Deployment of early diagnosis and mefloquine-artesunate treatment of falciparum malaria in Thailand: the Tak Malaria Initiative. PLoS Med. 2006;3:e183.

9. Zhou G, Sirichaisinthop J, Sattabongkot J, Jones J, Bjornstad ON, Yan G, et al. Spatio-temporal distribution of Plasmodium falciparum and P. vivax malaria in Thailand. Am J Trop Med Hyg. 2005;72:256-62.

10. Baum E, Sattabongkot J, Sirichaisinthop J, Kiattibutr K, Davies DH, Jain A, et al. Submicroscopic and asymptomatic Plasmodium falciparum and Plasmodium vivax infections are common in western Thailand - molecular and serological evidence. Malar J. 2015;14:95.

11. Imwong M, Nguyen TN, Tripura R, Peto TJ, Lee SJ, Lwin KM, et al. The epidemiology of subclinical malaria infections in South-East Asia: findings from cross-sectional surveys in Thailand-Myanmar border areas, Cambodia, and Vietnam. Malar J. 2015;14:381.

12. Buates S, Bantuchai S, Sattabongkot J, Han ET, Tsuboi T, Udomsangpetch $\mathrm{R}$, et al. Development of a reverse transcription-loop-mediated 
isothermal amplification (RT-LAMP) for clinical detection of Plasmodium falciparum gametocytes. Parasitol Int. 2010;59:414-20.

13. Aydin-Schmidt B, Xu W, Gonzalez IJ, Polley SD, Bell D, Shakely D, et al. Loop mediated isothermal amplification (LAMP) accurately detects malaria DNA from filter paper blood samples of low density parasitaemias. PLoS ONE. 2014;9:e103905.

14. Cook J, Aydin-Schmidt B, Gonzalez IJ, Bell D, Edlund E, Nassor MH, et al. Loop-mediated isothermal amplification (LAMP) for point-of-care detection of asymptomatic low-density malaria parasite carriers in Zanzibar. Malar J. 2015;14:43.

15. Hopkins H, Gonzalez IJ, Polley SD, Angutoko P, Ategeka J, Asiimwe C, et al. Highly sensitive detection of malaria parasitemia in a malaria-endemic setting: performance of a new loop-mediated isothermal amplification kit in a remote clinic in Uganda. J Infect Dis. 2013;208:645-52.

16. Sattabongkot J, Tsuboi T, Han ET, Bantuchai S, Buates S. Loop-mediated isothermal amplification assay for rapid diagnosis of malaria infections in an area of endemicity in Thailand. J Clin Microbiol. 2014;52:1471-7.

17. Sirichaisinthop J, Buates S, Watanabe R, Han ET, Suktawonjaroenpon W, Krasaesub S, et al. Evaluation of loop-mediated isothermal amplification (LAMP) for malaria diagnosis in a field setting. Am J Trop Med Hyg. 2011:85:594-6.

18. Liu H, Li XT, Hu B, Zhang L, Xue XH, Lv S, et al. Development of reverse transcription loop-mediated isothermal amplification for rapid detection of Batai virus in cattle and mosquitoes. Vector Borne Zoonotic Dis. 2016;16:415-22.

19. Cui L, Mascorro CN, Fan Q, Rzomp KA, Khuntirat B, Zhou G, et al. Genetic diversity and multiple infections of Plasmodium vivax malaria in Western Thailand. Am J Trop Med Hyg. 2003;68:613-9.

20. Baum E, Sattabongkot J, Sirichaisinthop J, Kiattibutr K, Jain A, Taghavian $\mathrm{O}$, et al. Common asymptomatic and submicroscopic malaria infections in Western Thailand revealed in longitudinal molecular and serological studies: a challenge to malaria elimination. Malar J. 2016;15:333.

21. Moody A. Rapid diagnostic tests for malaria parasites. Clin Microbiol Rev. 2002;15:66-78.

22. Han ET, Watanabe R, Sattabongkot J, Khuntirat B, Sirichaisinthop J, Iriko $\mathrm{H}$, et al. Detection of four Plasmodium species by genus- and speciesspecific loop-mediated isothermal amplification for clinical diagnosis. J Clin Microbiol. 2007:45:2521-8.

23. Lau YL, Fong MY, Mahmud R, Chang PY, Palaeya V, Cheong FW, et al. Specific, sensitive and rapid detection of human Plasmodium knowles infection by loop-mediated isothermal amplification (LAMP) in blood samples. Malar J. 2011;10:197.
24. Kimura M, Kaneko O, Liu Q, Zhou M, Kawamoto F, Wataya Y, et al. Identification of the four species of human malaria parasites by nested PCR that targets variant sequences in the small subunit rRNA gene. Parasitol Int. 1997:46:91-5.

25. Singh B, Bobogare A, Cox-Singh J, Snounou G, Abdullah MS, Rahman HA. A genus-and species-specific nested polymerase chain reaction malaria detection assay for epidemiologic studies. Am J Trop Med Hyg. 1999;60:687-92.

26. Singh B, Kim Sung L, Matusop A, Radhakrishnan A, Shamsul SS, Cox-Singh $J$, et al. A large focus of naturally acquired Plasmodium knowlesi infections in human beings. Lancet. 2004;363:1017-24.

27. WHO. World malaria report 2016. Geneva: World Health Organization; 2016.

28. Department of Disease Control. Weekly report malaria situation in Thailand 2017. Nonthaburi: Ministry of Public Health; 2017.

29. Nguitragool W, Mueller I, Kumpitak C, Saeseu T, Bantuchai S, Yorsaeng R, et al. Very high carriage of gametocytes in asymptomatic low-density Plasmodium falciparum and P. vivax infections in western Thailand. Parasit Vectors. 2017;10:512.

30. Phommasone K, Adhikari B, Henriques G, Pongvongsa T, Phongmany P, von Seidlein $L$, et al. Asymptomatic Plasmodium infections in 18 villages of southern Savannakhet Province, Lao PDR (Laos). Malar J. 2016;15:296.

31. Tripura R, Peto TJ, Veugen CC, Nguon C, Davoeung C, James N, et al. Submicroscopic Plasmodium prevalence in relation to malaria incidence in 20 villages in western Cambodia. Malar J. 2017;16:56.

32. Laishram DD, Sutton PL, Nanda N, Sharma VL, Sobti RC, Carlton JM, et al. The complexities of malaria disease manifestations with a focus on asymptomatic malaria. Malar J. 2012;11:29.

33. Djimde AA, Doumbo OK, Traore O, Guindo AB, Kayentao K, Diourte Y, et al. Clearance of drug-resistant parasites as a model for protective immunity in Plasmodium falciparum malaria. Am JTrop Med Hyg. 2003;69:558-63.

34. Doolan DL, Dobano C, Baird JK. Acquired immunity to malaria. Clin Microbiol Rev. 2009:22:13-36.

35. Zaw MT, Thant M, Hlaing TM, Aung NZ, Thu M, Phumchuea K, et al. Asymptomatic and sub-microscopic malaria infection in Kayah State, eastern Myanmar. Malar J. 2017;16:138
Ready to submit your research? Choose BMC and benefit from:

- fast, convenient online submission

- thorough peer review by experienced researchers in your field

- rapid publication on acceptance

- support for research data, including large and complex data types

- gold Open Access which fosters wider collaboration and increased citations

- maximum visibility for your research: over $100 \mathrm{M}$ website views per year

At BMC, research is always in progress.

Learn more biomedcentral.com/submissions 